15 THE ROLE OF TRUST IN SENIOR LEADERS' EXPERIENCES OF USING ANALYTICS TO INFORM STRATEGIC HEALTH AND CARE DECISIONS

${ }^{1} E$ Ingram, 'S Beardon, 'S Cooper, ${ }^{2} \mathrm{D}$ Osborn, ${ }^{1} \mathrm{M}$ Gomes, ${ }^{3} \mathrm{H} \mathrm{McD}$ onald, ${ }^{4} \mathrm{~S}$ Hogarth, IJ Sheringham. ${ }^{1}$ Department of Applied Health Research, UCL, UK; ${ }^{2}$ Division of Psychiatry, UCL, UK; ${ }^{3}$ NIHR Health Protection Research Unit (HPRU) in Immunisation, LSHTM; ${ }^{4}$ London Boroughs of Camden and Islington

\subsection{6/bmjopen-2021-QHRN.15}

Senior leaders make strategic decisions about the structure and delivery of health and care services. Analysis of pseudonymised administrative data from residents' health or care records (analytics) aims to support high-quality strategic decisions, but its impacts depend on how analytics are perceived and used by senior decision-makers.

Aim or objectives To understand how the process of generating knowledge from analytics could be enhanced for those making strategic health and care decisions.

Methods Individual semi-structured interviews were conducted with 20 senior decision-makers from constituent organisations of North Central London's Sustainability and Transformation Partnership. Interviews were analysed using the Framework Method.

Results Trust emerged as a concept in participants' experiences of using analytics in three key ways: data quality and accuracy; trust in sharing data for analytics; and trust between decision-makers. Firstly, participants expressed concerns around data quality and these shaped decision-making processes as some made decisions based on anecdotal information or gut feeling instead of untrusted data. Others sought 'more accurate' data, whilst some decisions were 'stopped in their tracks' because of a lack of trust in available data. Secondly, participants' ability to obtain data was influenced by others' willingness to share, itself driven by levels of trust in how that data will be subsequently analysed and used. Finally, a lack of trust between key leaders across health and care led to concerns that decisions based on analytics may act against individual organisational priorities and interests. Where personal trust between individuals across organisations existed, there was greater scope for decisions across organisational boundaries to be informed by analytics.

Conclusions Trust is a key component of how senior leaders approach and use analytics. Developing trust in data quality and between leaders could influence if, when and how leaders obtain and use analytics to inform strategic health and care decisions.

\section{IT TAKES A COMMUNITY TO MAKE AN EXPERT: EXPLORING PROFESSIONAL EXPERTISE AMONGST ALLIED HEALTH PROFESSIONALS}

${ }^{1} \mathrm{~B}$ Jackson, ${ }^{1} \mathrm{~S}$ Purdy, ${ }^{2} \mathrm{H}$ Cooper-Thomas. ${ }^{1}$ University of Auckland, New Zealand; ${ }^{2} \mathrm{AUT}$, Auckland, New Zealand

\subsection{6/bmjopen-2021-QHRN.16}

Background The current healthcare environment provides several challenges to the existing roles of healthcare professionals and the value of the professional expert is under scrutiny. The study of professional experts can cast light on performance that is above and beyond core competency. The study aimed to generate an understanding of professional experts amongst practitioners in the allied health community in New Zealand (NZ).

Method An online survey gathered opinions about professional experts in the community $(n=119)$. Forty-five highly-experienced allied health practitioners (AHPs) currently practicing in NZ were interviewed. This mixed method study explored critical incident narratives that highly experienced practitioners told about their work. From four investigations, findings are brought together to inform what it might take to become known as an expert.

Findings Thematic analysis generated two interconnected themes of a professional expert being a personal leader and teacher, as well as a highly experienced, knowledgeable and skilful practitioner. Participants clearly identified being 'highly experienced' and 'having in-depth knowledge' as essential elements of professional expertise, but also valued other characteristics highly. Affirming, challenging and otherwise transformative incidents formed a backdrop to detailed narrative study that highlighted the practitioners' search for meaning and its role in the development and expression of expert performance. Narrative analysis indicated that professional confidence and expertise are intrinsically linked and could be explicitly developed, through knowledge sharing, reflective practices and narrative reframing. The community has an essential role in developing and encouraging experts to emerge.

Conclusion This study presents a novel construction of professional experts and expertise amongst practitioners in a current healthcare community. Understanding how professional experts and their expertise is constructed within a community can provide practitioners, educators and leaders with new insights that may alter the opportunities and supports provided for attaining expertise.

\section{BARRIERS AND FACILITATORS FOR IMPLEMENTATION OF A COMPUTERIZED CLINICAL DECISION SUPPORT SYSTEM IN LUNG CANCER MULTIDISCIPLINARY TEAM MEETINGS - A QUALITATIVE ASSESSMENT}

${ }^{1} \mathrm{~S}$ Klarenbeek, ${ }^{2} \mathrm{O}$ Schuurbiers-Siebers, ${ }^{2} \mathrm{M}$ van den Heuvel, ${ }^{1} \mathrm{M}$ Prokop, ${ }^{3} \mathrm{M}$ Tummers ${ }^{1}$ Radboud UMC, Department of Medical Imaging, Netherlands; ${ }^{2}$ Radboud UMC, Department of pulmonary diseases, Netherlands; ${ }^{3}$ Radboud UMC, Department of Health Evidence, Netherlands

\subsection{6/bmjopen-2021-QHRN.17}

Background Oncological computerized clinical decision support systems to facilitate workflows of multidisciplinary team meetings (MDTMs) are currently being developed. To reach successful implementation of these CDSSs in MDTMs, this study aims to: a) identify barriers and facilitators for successful implementation for the use in case of lung cancer; and b) provide actionable findings for an implementation strategy.

Methods The Consolidated Framework for Implementation Science was used to create an interview protocol for CDSSs in lung cancer MDTMs and to analyze the results. Semi-structured interviews were conducted among various health care professionals involved in these MDTMs. The transcripts were analyzed using a thematic analysis following a deductive approach. 
Results A total of 26 professionals participated in the interviews. The main facilitator for implementation of the CDSS was considered to be easy access to well-structured patient data, and the resulting reduction of MDTM preparation time and of duration of MDTMs. Less impact of the CDSS was expected on the quality of lung cancer services generated by MDTM decision-making. Main barriers for adoption included incomplete or non-trustworthy output generated by the system and insufficient adaptability of the system to local and contextual needs. Actionable findings for an implementation strategy were a usability test involving key users and a validation study in the organization's real-life setting prior to roll out.

Conclusion Using this CDSS in lung cancer MDTMs was expected to increase efficiency of workflows. Successful implementation is dependent on the reliability and adaptability of the CDSS and involvement of key users in the implementation process.

\section{COLLABORATIVE QUALITATIVE RESEARCH ON SUICIDE AND SELF-HARM IN SOUTH ASIA: A REFLECTION ON CHALLENGES AND SOLUTIONS}

${ }^{1}$ A Krayer, ${ }^{2} \mathrm{~S}$ Das. ${ }^{1}$ School of Health Sciences, Bangor University, UK; ${ }^{2}$ SASHI project, Mysore, India

\subsection{6/bmjopen-2021-QHRN.18}

Trust is essential to planning and delivering impactful international research that is culturally appropriate and has the potential to change practice and policy on local levels. However, details on how this is can be achieved, and a discussion of challenges encountered are often lacking. A better understanding of building and maintaining of trust in North-South research partnerships is essential, especially when tackling complex and sensitive issues such as self harm and suicide. Suicide is amongst the leading causes of death in South Asia.

This talk will reflect on experiences in the South Asia SelfHarm Initiative (SASHI), a global-challenges funded research project, led by co-investigators from the Global North and South. The research collects empirical evidence to inform the understanding of the nature of self-harm in the context of profound social, political and economic challenges in the global South as well as builds research capacity. We draw on Ben-Ari and Enosh's work (2010), which focuses on identifying incongruities that challenge our knowledge (discovery) and examine them in-depth as a source of new knowledge (construction) to come to a new understanding. The definition of trust is debated, and our starting point is Luhmann's (1999) approach that trust is expressed through social action in contexts we cannot fully know.

We argue that trust is a building block for fair and equitable international research partnerships and is continually developed and negotiated in relationships and activities. Power inequalities and contextual factors need to be acknowledged. Working on building and maintaining trust is emotionally and cognitively challenging. Our experiences suggest that building and maintaining trust relies on recognising similarities, which can foster respect and equality of status. Acknowledging and exploring differences can provide opportunities for reflection and joint learning. These issues are important to consider as they ultimately shape knowledge production and translation.
THE DYNAMICS OF TRUST AND STRUCTURAL COERCION WITHIN A MENINGITIS TRIAL IN SUB-SAHARAN AFRICA

${ }^{1} \mathrm{D}$ Lawrence, ${ }^{2} \mathrm{~A}$ Ssali, ${ }^{3} \mathrm{~K}$ Tsholo, ${ }^{1} \mathrm{~J}$ Jarvis, ${ }^{1} \mathrm{~J}$ Seeley. 'London School of Hygiene and Tropical Medicine, UK; ${ }^{2}$ MRC/UVRI and LSHTM Uganda Research Institute, Uganda; ${ }^{3}$ Botswana Harvard AIDS Institute Partnership, Botswana

\subsection{6/bmjopen-2021-QHRN.19}

Background Clinical trials in sub-Saharan Africa typically offer better medical care than is routinely available. This can lead to structural coercion where an individual may consent because of a lack of alternative options and potentially despite being uncertain about the research. An inherent component of this decision making process is an assessment of trust. Trust in the treatment options, the research team, and the process as a whole. This may be polarised in the context of life-threatening illnesses where recruitment (or not) could determine survival.

Aim We sought to understand the dynamics of trust and structural coercion in a multi-site clinical trial for HIV-associated cryptococcal meningitis.

Methods We embedded an ethnographic study within a clinical trial for HIV-associated cryptococcal meningitis. We conducted in-depth interviews with trial participants and their next-of-kin in Uganda and Botswana. We combined these with direct observations and in-depth interviews with researchers working at the African sites and European partner institutions. Interviews were transcribed, translated, and subject to narrative analysis.

Results To date we have recruited 14 trial participants, five next-of-kin and ten researchers. Recruitment is on-going until March 2021. Participants and their relatives often felt they had no choice but to enrol in the clinical trial which was their best chance of survival. Despite the perceived benefits of participation, recruitment came at a cost to participants who agreed to invasive medical procedures such as lumbar punctures despite pre-existing beliefs they could cause death. The severity of the illness contributed to poor comprehension of what the trial entailed and the decision to participate was heavily based on trust in the research team.

Conclusions Structural coercion is a significant factor impacting recruitment into clinical trials in resource-limited settings. In the context of life-threatening illness, trust superseded the need for an in-depth understanding of the research process.

\section{TRUST AND RELIANCE WITHIN SPECIALIST CLINICAL SERVICES: COUNTER-PRODUCTIVE OR HELPFUL FOR SELF-MANAGEMENT OF PEOPLE WITH NEUROMUSCULAR CONDITIONS?}

${ }^{1}$ LE Lee, ${ }^{2}$ ST Kulnik, ${ }^{2}$ A Boaz, ${ }^{3} \mathrm{G}$ Ramdharry. 'Department of Neuromuscular Diseases, UCL Queen Square Institute of Neurology, UK; ${ }^{2}$ Faculty of Health Social Care and Education, Kingston University and St George's University of London, UK; ${ }^{3}$ Department of Neuromuscular Diseases, UCL Queen Square Institute of Neurology, UK

\subsection{6/bmjopen-2021-QHRN.20}

Current approaches to self-management de-emphasise dependency on healthcare services and focus on building confidence and capability. Our qualitative study explores self-management perspectives from individuals with neuromuscular conditions who attend regional specialist clinics, to inform implementation of a self-management intervention. 